\title{
Deronegative Celiac Disease; Frequently Encountered Yet Undiagnosed Clinical Entity
}

\author{
Kapil Dhingra ${ }^{1}$, Sudhir Maharshi ${ }^{2, *}$, Bharat Sapra ${ }^{3}$, Sandeep Ratra ${ }^{1}$, Shyam Sunder Sharma ${ }^{3}$, \\ Sandeep Nijhawan ${ }^{3}$
}

1. Senior Resident, Department of Gastroenterology, SMS Medical College and Hospitals, Jaipur, India

2. Associate Professor, Department of Gastroenterology, SMS Medical College and Hospitals, Jaipur, India

3. Senior Professor, Department of Gastroenterology, SMS Medical College and Hospitals, Jaipur, India

* Corresponding Author:

Sudhir Maharshi, MD

Associate Professor, Department of

Gastroenterology, Room No. 303, SMS

Hospital, Jaipur, Rajasthan 302004, India

Tel: + 911412518291

Fax: + 911412564222

Email: sudhir.maharshi@gmail.com

Received: 22 May 2020

Accepted: 28 Oct. 2020

\section{ABSTRACT}

\section{BACKGROUND}

There are limited studies on the seronegative celiac disease from the Indian subcontinent. The aim of this study was to assess the prevalence, pathological, genetic, and clinical profile of patients with seronegative celiac disease.

\section{METHODS}

This prospective observational study was conducted in the Department of Gastroenterology, SMS Hospital, Jaipur, between October 2017 to March 2019. Consecutive patients with seronegative celiac disease with age $\geq 3$ years were enrolled for the assessment of demography, clinical features, histological findings, celiac serology, genetic analysis, and response to gluten-free diet.

\section{RESULTS}

Out of total of 312 patients with celiac disease, 13 (4.16\%) patients (median age 25 years [range 5-46 years], 10 female) were diagnosed as having seronegative celiac disease. Presenting symptoms were chronic diarrhea in nine $(69.23 \%)$, abdominal pain in six $(46.15 \%)$, weight loss in five $(38.46 \%)$, and short stature in two $(15.38 \%)$ patients. On histological analysis, Marsh stage 2 was seen in five $(38.46 \%)$, Marsh $3 \mathrm{c}$ in two (15.38\%), Marsh 3a in three (23.07\%), and Marsh 3b in three (23.07\%) patients. On HLA analysis, HLA-DQ2.5 was seen in six $(46.15 \%)$ patients, HLA-DQ2.2 in five (38.46\%), and HLA-DQ8 in two $(15.38 \%)$ patients.

\section{CONCLUSION}

The prevalence of seronegative celiac disease in our study was $4.16 \%$. The most common symptoms were chronic diarrhea and abdominal pain, and the histological grade was Marsh stage 2 .

\section{KEYWORDS:}

Celiac disease, Clinical intestinal disorders, Diarrhea and malabsorption

Please cite this paper as:

Dhingra K, Maharshi S, Sapra B, Ratra S, Sharma SS, Nijhawan S. Seronegative Celiac Disease; Frequently Encountered Yet Undiagnosed Clinical Entity. Middle East J Dig Dis 2021;13:35-42. doi: 10.34172/mejdd.2021. 201. 


\section{INTRODUCTION}

Celiac disease (CD) is an autoimmune enteropathy characterized by villous atrophy and lymphocytic inflammation of the epithelial layer covering the mucosa in genetically susceptible subjects. ${ }^{1}$ The diagnosis of seropositive CD is based on the findings of positive antibodies (anti-tissue transglutaminase [tTG] or endomysial antibody [EMA]) in combination with histological changes (increased intraepithelial lymphocytes or villous atrophy) in duodenal biopsies. $^{2}$ The recommended antibody is IgA anti-tTG as a screening test due to its high sensitivity and specificity. Although CD antibodies are detected in most of the patients with CD (sensitivity $95 \%$ to $98 \%$ ), a minority of patients with CD may have negative serology, and in these cases, the diagnosis depends on typical histopathology along with HLA-DQ2 and/or HLA-DQ8 positivity, which is a mandatory requirement to suspect the diagnosis of seronegative celiac disease (SNCD). ${ }^{3,4}$ Furthermore, clinical, laboratory, and histological improvement should be proved after an adequate period (6-24 months) of a gluten-free diet (GFD) because a subgroup of patients with irritable bowel syndrome may have symptomatic improvement with GFD. ${ }^{5}$ The duodenal histopathology compatible with CD having negative serology is a clinical challenge since such histopathological changes can be observed in a variety of diseases other than $\mathrm{CD}$, including autoimmune enteropathy, common variable immunodeficiency (CVID), parasitic infection (Giardia lamblia), Crohn's disease, tropical sprue, eosinophilic gastroenteritis, drug-induced enteropathy (mainly due to angiotensin II inhibitors), intestinal lymphoma, human immunodeficiency virus (HIV) infection, and Whipple's disease. ${ }^{5-13}$ Thus, prior to making a firm diagnosis of SNCD, it is mandatory to rule out other causes of villous atrophy in order to avoid an unnecessary lifelong GFD. The reported prevalence of SNCD ranges from $1.03 \%$ in all patients with CD to $28 \%$ in patients with latent CD. ${ }^{14,15}$ Regarding the pathogenesis of SNCD; it has been speculated that the seronegativity in these patients is because of deposition of immune complex deep into intestinal lamina propria, which does not reach the blood vessels. ${ }^{16}$ Immaturity of plasma cells is an alternative hypothesis in the mechanism of SNCD. ${ }^{10,17}$ Patients with SNCD may have a poor prognosis and a high risk of developing complications. ${ }^{18-19}$ There is limited data on SNCD from the Indian subcontinent. Our study was designed to evaluate the prevalence, and pathological, genetic, and clinical profile of patients with SNCD.

\section{MATERIALS AND METHODS}

This prospective observational study was conducted at SMS Hospital, a tertiary care center in Jaipur, India, between October 2017 to March 2019. Written informed consent was obtained from each patient, and the study protocol, conformed to the ethical guidelines of the 1975 declaration of Helsinki as reflected in priori, was approved by the appropriate institutional review committee. All consecutive patients with clinical signs and symptoms of CD were tested for IgA anti tTG antibody and underwent upper gastrointestinal endoscopy for duodenal biopsy. Patients with positive antibodies and biopsy consistent with $\mathrm{CD}$ were labeled as seropositive CD. Those patients with negative serology and biopsy were ruled out and evaluated for other causes for their symptoms. Patients with negative serology and histology changes on biopsy suggestive of $\mathrm{CD}$ were tested for total IgA levels to rule out IgA deficiency and if they were found IgA deficient, were further tested for IgG anti tTG and if it was positive, patients were labeled as seropositive CD. Patients with normal IgA levels were tested for human leukocyte antigen (HLA) haplotypeDQ2/DQ8. If the patient was negative for HLA, the $\mathrm{CD}$ was ruled out. If found positive, other causes of seronegative villous atrophy were ruled out, and patients were subjected to GFD. Response to GFD was assessed at 6 months by the clinical, laboratory, and histological improvement. Those patients who responded to GFD were labeled as having SNCD. The approach to diagnose SNCD is shown in figure 1. Seronegative negative celiac was diagnosed in patients on the basis of clinical features, proximal small intestinal biopsies compatible to $\mathrm{CD}$ according to Marsh classification and positive for HLA-DQ2 and/or DQ8 alleles but negative for serology markers. Histological changes compatible with CD range from inflammation to villous atrophy and were stratified according to Marsh-Oberhuber classification. ${ }^{13,20,21}$ All the patients with SNCD who aged $>3$ years attending our department were included in the study. Patients with parasitic infection (Giardia lamblia), autoimmune enteropathy, CVID, eosinophilic gastroenteritis, druginduced enteropathy (mainly due to angiotensin II in- 


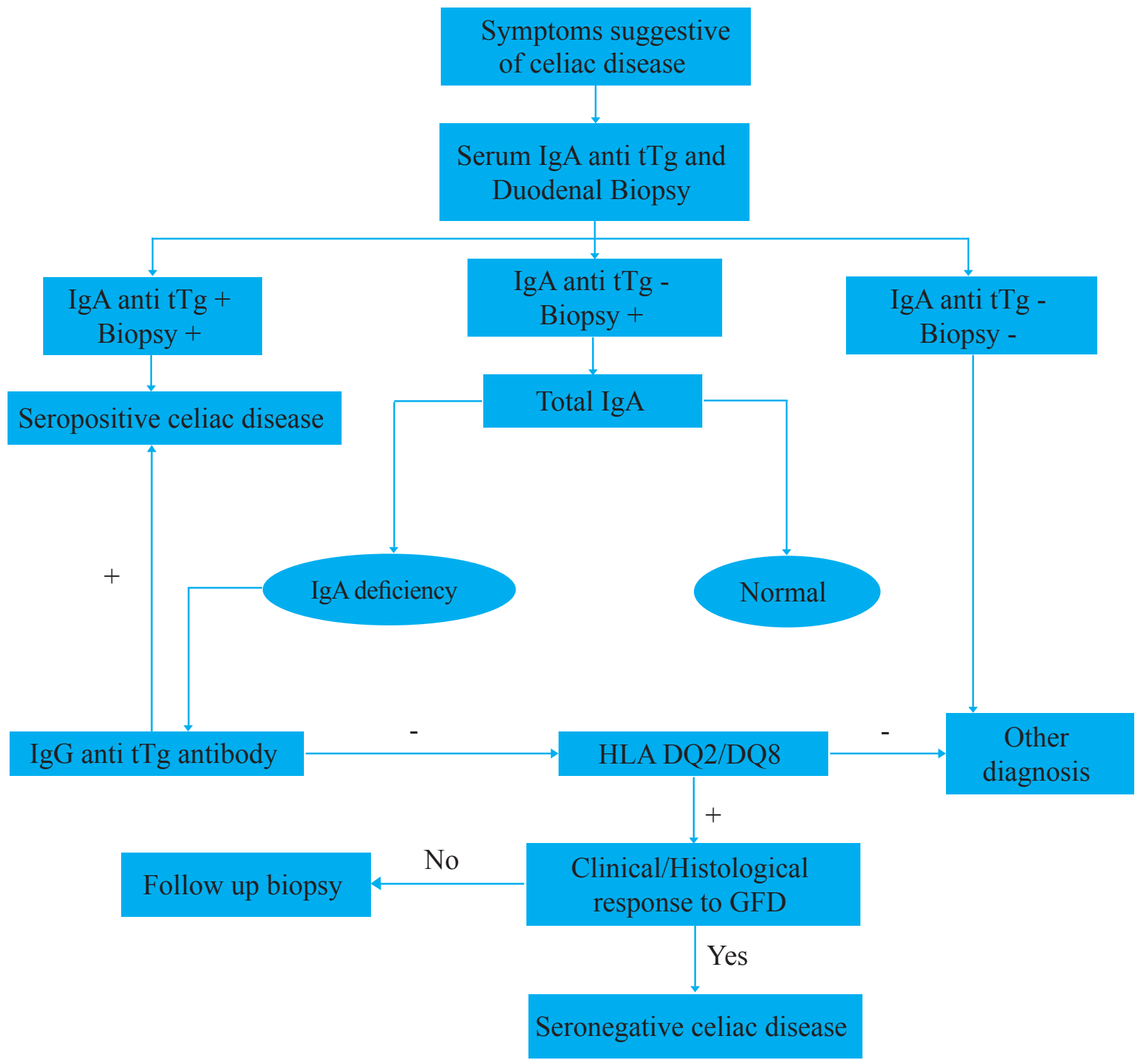

Fig.1: Flowchart showing approach for the diagnosis of seronegative celiac disease

hibitors), intestinal lymphoma, Crohn's disease, tropical sprue, HIV infection, and Whipple's disease were excluded. A detailed history was obtained from each patient, along with clinical examination. Laboratory tests included complete and differential blood count, serum iron, ferritin, vitamin B12, and folic acid levels, HIV ELISA, thyroid functions test, serum immunoglobulins, liver function tests, and random blood sugar levels. Stool samples were examined for ova, bacteria, and parasites, and stool antigen test for giardia. Serology markers for CD included IgA anti tTG. In cases with selective IgA deficiency, IgG anti tTG was assayed. The CD serology tests were done by a validated, standardized, and reliable commercial kit by enzyme immunoassay method (CHORUS, Siena, Italy). Each patient underwent upper gastrointestinal endoscopy, and at least 6 biopsy samples were taken from the second part of the duodenum. The biopsies were interpreted by one senior histopathologist. Findings were expressed according to the modified Marsh classification in CD.22A detailed HLA typing including HLA-DQ2.5 (DQA1*0501, DQB1*0201), HLA-DQ8(DQA1*03, 
Table 1: Demographic, pathological, genetic, and clinical profile of the patients with SNCD $(n=13)$

\begin{tabular}{lc}
\hline Median age (years) with range & $25(5-46)$ \\
\hline Sex & $\mathbf{n}(\%)$ \\
Male & $3(23.07)$ \\
\hline Female & $10(76.93)$ \\
\hline Clinical features & $\mathbf{n}(\%)$ \\
Chronic diarrhea & $9(69.23)$ \\
\hline abdominal pain & $6(46.15)$ \\
\hline Weight loss & $5(38.46)$ \\
\hline Short stature & $2(15.38)$ \\
\hline Anemia & $6(46.15)$ \\
\hline Associated autoimmune diseases & $\mathbf{n ~ ( \% )}$ \\
Hypothyroidism & $2(15.38)$ \\
\hline Histology n (\%) & \\
\hline Marsh 2 & $5(38.46)$ \\
\hline Marsh 3a & $3(23.07)$ \\
\hline Marsh 3b & $3(23.07)$ \\
\hline Marsh 3c & $2(15.38)$ \\
\hline HLA analysis & $\mathbf{n}(\%)$ \\
HLA DQ 2.5 & $6(46.15)$ \\
\hline HLA DQ 2.2 & $5(38.46)$ \\
\hline HLA DQ 8 & $2(15.38)$ \\
\hline SNCD- Seronegative celiac disease, HLA- Human leucocyte antigen)
\end{tabular}

DQB1*0302), HLA-DQ 2.2 (DQA1*0201, DQB1*0202), and HLA-DQ7.5 (DQA1*05, DQB1*0301) was performed by polymerase chain reaction (PCR) in cases with a discrepancy between histology and serology.

\section{Statistical analysis}

The statistical analysis was performed using statistical package for social sciences (SPSS) trial version 23.0 for windows (SPSS, Chicago IL, USA). Data were expressed as an absolute number and percentage for categorical variables and median and ranges for continuous variables, as appropriate. Wilcoxon's signed-rank test and MannWhitney $U$ test were used to analyze paired and unpaired data, respectively. A 'p' value of $<0.05$ was considered to be significant.

\section{RESULTS}

A total of 312 patients with $\mathrm{CD}$ were studied during the study period; of them, 13 (4.16\%) patients were diagnosed as having SNCD. The median age of the patients was 25
Table 2: Laboratory parameters at baseline and 6 months after GFD of patients with SNCD

\begin{tabular}{|c|c|c|}
\hline $\begin{array}{l}\text { Parameters - } \\
\text { Median (IQR) }\end{array}$ & Baseline & $\begin{array}{c}6 \text { months after } \\
\text { GFD }\end{array}$ \\
\hline Hemoglobin (g/dL) & $10.75(9.07-11.85)$ & $12.50(10.90-12.95)^{*}$ \\
\hline MCV (fL/red cell) & $76.1(64.33-85.38)$ & $82.2(73.42-88.52)^{*}$ \\
\hline $\begin{array}{l}\text { Total leucocyte } \\
\text { count (cells/cumm) }\end{array}$ & $7.5(5.7-8.9)$ & $7.8(5.6-10.2)$ \\
\hline Ferritin (ng/mL) & $45(10-70)$ & $51(21-88)$ \\
\hline Iron $(\mu \mathrm{g} / \mathrm{dL})$ & $90(50-110)$ & $96(56-124)$ \\
\hline $\begin{array}{l}\text { Vitamin B12 } \\
(\mathrm{pg} / \mathrm{mL})\end{array}$ & $389(300-460)$ & $396(310-480)$ \\
\hline $\begin{array}{l}\text { Folic acid } \\
(\mathrm{ng} / \mathrm{mL})\end{array}$ & $16(9-20)$ & $23(13-28)^{*}$ \\
\hline $\begin{array}{l}\text { Blood sugar } \\
(\mathrm{mg} / \mathrm{dL})\end{array}$ & $98(89-111)$ & $102(92-124)$ \\
\hline Albumin(mg/dL) & $3.4(3.2-3.9)$ & $3.5(3.2-4.1)$ \\
\hline $\begin{array}{l}\text { Serum bilirubin } \\
(\mathrm{mg} / \mathrm{dL})\end{array}$ & $0.89(0.78-1.01)$ & $0.92(0.74-1.04)$ \\
\hline $\begin{array}{l}\text { Aspartate amino- } \\
\text { transferase (IU/L) }\end{array}$ & $34(15-42)$ & $32(13-41)$ \\
\hline $\begin{array}{l}\text { Alanine amino- } \\
\text { transferase (IU/L) }\end{array}$ & $38(14-46)$ & $36(15-43)$ \\
\hline
\end{tabular}

years (range 5-46 years) with female predominance (10 females $[\mathrm{F}], 3$ males $[\mathrm{M}] ; \mathrm{F} / \mathrm{M}=3.3 / 1)$. The baseline demographic, pathological, genetic, and clinical parameters of patients with SNCD are shown in table 1. All patients with SNCD had histological alterations in small intestinal mucosa consistent with CD and positive HLA analysis. Most of the patients with SNCD reported gastrointestinal symptoms. The most common presenting feature was chronic diarrhea, as seen in nine $(69.23 \%)$ patients. Six (46.15\%) patients had abdominal pain, five (38.46\%) had weight loss, and two (15.38\%) had short stature. Anemia was present in six $(46.15 \%)$ patients, five had iron deficiency, and one had megaloblastic anemia. Associated hypothyroidism was observed in two (15.38\%) patients. HLA analysis disclosed positivity for HLADQ2.5 in six (46.15\%) patients, HLA-DQ2.2 in five (38.46\%), and HLA-DQ8 in two (15.38\%) patients, as shown in table 1 . None of the patients had a family history of $\mathrm{CD}$. Baseline and 6 months post GFD laboratory parameters of patients with SNCD are shown in table 2. There was a significant improvement in hemoglobin, mean corpuscular volume (MCV), and serum folic acid level 6 months after GFD in study patients. On histological analysis, 
Table 3: Clinical and histological features of individual study patient with SNCD before and 6 months after GFD

\begin{tabular}{|c|c|c|c|c|c|}
\hline Number & Age (in years)/Sex & Clinical features & $\begin{array}{c}\text { Pretreatment } \\
\text { duodenal biopsy }\end{array}$ & $\begin{array}{c}\text { Symptomatic } \\
\text { response to GFD }\end{array}$ & $\begin{array}{c}\text { Follow-up duode- } \\
\text { nal biopsy }\end{array}$ \\
\hline 1 & $44 / \mathrm{F}$ & $\mathrm{D}, \mathrm{P}$ & Marsh 2 & Non-compliant & - \\
\hline 2 & $16 / \mathrm{F}$ & $\mathrm{D}, \mathrm{WL}$ & Marsh 3c & $\begin{array}{l}\text { D improved, } \\
\text { weight gain }\end{array}$ & Refused \\
\hline 3 & $30 / \mathrm{F}$ & $\mathrm{P}, \mathrm{WL}$ & Marsh 2 & Weight gain & Normal \\
\hline 4 & $5 / \mathrm{M}$ & $\mathrm{D}, \mathrm{S}$ & Marsh 3b & D improved & Marsh 2 \\
\hline 5 & $30 / \mathrm{F}$ & $\mathrm{D}, \mathrm{WL}$ & Marsh 3b & D improved & Normal \\
\hline 6 & $25 / \mathrm{F}$ & $\mathrm{P}$ & Marsh 3a & Non-compliant & - \\
\hline 7 & $5 / F$ & S, D & Marsh 2 & D improved & Refused \\
\hline 8 & $23 / \mathrm{F}$ & $\mathrm{P}, \mathrm{WL}$ & Marsh 3c & Weight gain & Marsh 1 \\
\hline 9 & $23 / \mathrm{F}$ & D & Marsh 3a & D improved & Normal \\
\hline 10 & $30 / \mathrm{F}$ & $\mathrm{P}$ & Marsh 2 & Non-compliant & - \\
\hline 11 & $46 / \mathrm{F}$ & $\mathrm{D}, \mathrm{P}$ & Marsh 3a & D improved & Normal \\
\hline 12 & $29 / \mathrm{M}$ & $\mathrm{D}$ & Marsh 2 & D improved & Normal \\
\hline 13 & $18 / \mathrm{M}$ & $\mathrm{D}, \mathrm{WL}$ & Marsh 3b & D improved & Marsh 2 \\
\hline
\end{tabular}

(F: Female, M: Male, D: Chronic diarrhea, P: Pain abdomen, S: Short stature, WL: Weight loss)

Table 4: Demographic and clinical profile of patients with SNCD and SPCD

\begin{tabular}{lccc}
\hline Parameters & SNCD patients $(\mathbf{n}=\mathbf{1 3})$ & SPCD patients $(\mathbf{n}=\mathbf{2 9 9})$ & $\boldsymbol{p}$ value \\
\hline Median age (years) with range & $25(5-46)$ & $31(6-64)$ & 0.88 \\
\hline Sex (Male:Female ratio) & $1: 3.3$ & $1: 4.2$ & 0.90 \\
\hline $\begin{array}{l}\text { Clinical features n (\%) } \\
\begin{array}{l}\text { Classical features (diarrhea, pain abdomen, anemia and } \\
\text { weight loss) }\end{array}\end{array}$ & $13(100)$ & $146(48.8 \%)$ & 0.01 \\
\hline Short stature & $2(15.38)$ & $51(17.05)$ & 0.82 \\
\hline Associated autoimmune diseases n (\%) & $2(15.38)$ & $62(20.73)$ & 0.58 \\
\hline Total villous atrophy & $2(15.38)$ & $95(31.77)$ & 0.02 \\
\hline
\end{tabular}

(SNCD: Seronegative celiac disease, SPCD: Seropositive celiac disease)

five (38.46\%) patients had Marsh 2, three (23.07\%) patients had Marsh 3a, three (23.07\%) patients Marsh 3b, and two (15.38\%) patients had Marsh 3c stage of celiac involvement. All the study patients were advised GFD along with vitamin supplements. Out of the 13 patients, three were non-compliant to GFD, and their symptoms persisted. The remaining 10 patients were available for follow-up after a median follow-up of 7 months (range-6.5 to 8.5 months). Out of these 10 patients, eight gave consent for follow-up duodenal biopsy and their histology improved compared with pretreatment biopsy along with clinical improvement, as shown in table 3. The remaining two patients who did not give consent for follow-up duodenal biopsy experienced clinical improvement after 6 months on GFD. The detailed clinical and histological features of the individual patients with SNCD before and after GFD are shown in table 3. The demographic, and clinical profile of both SNCD and seropositive patients are shown in table 4 .

\section{DISCUSSION}

In our study on $312 \mathrm{CD}$ patients, the prevalence of SNCD was $4.16 \%$. The most common presenting symptom was chronic diarrhea followed by abdominal pain, weight loss, and short stature. Anemia was observed in six $(46.15 \%)$ patients and associated hypothyroidism in two $(15.38 \%)$ patients. On histological analysis, all the patients had features of $\mathrm{CD}$, and the most common stage was Marsh 2 observed in five (38.46\%) patients. On HLA analysis, the most common was HLA-DQ2.5, 
seen in six (46.15\%) patients, followed by HLA-DQ2.2 in five (38.46\%), and HLA-DQ8 in two (15.38\%) patients. To the best of our knowledge, this is the first study from north India on the prevalence, pathological, genetic, and clinical profile of SNCD. In a country like India, especially the northern part, where the prevalence of $\mathrm{CD}$ is high $(1.23 \%)$, clinicians frequently encounter patients who present with features of $\mathrm{CD}$ yet have normal titers of routinely done screening tests (IgA anti tTG and/or EMA). ${ }^{23}$ In these situations, where there is a high suspicion, there is a need to establish the diagnosis with further testing, including HLA analysis. Rapid and definite diagnosis of $\mathrm{CD}$ in such a group of patients leads to prompt clinical improvement and aversion of complications later in life. We report a prevalence of $4.16 \%$ of SNCD in our study patients. The existence of SNCD was previously reported in several studies, although its prevalence is still a matter of debate. ${ }^{4,15,24-26}$ Rostami and colleagues reported a high prevalence $(24 \%)$ of SNCD, while Abrams and co-workers reported a $15 \%$ prevalence of villous atrophy in patients negative for all CD-related serological tests ( $\operatorname{IgA}$ and $\operatorname{IgG}$ tTG, EMA and antigliadin antibody). Their study concluded that clinical features and mode of presentation of CD (classic or silent) were similar in seronegative and seropositive $\mathrm{CD}$, and they both improved following a GFD. ${ }^{4,24}$ In contrast, a recent study on pediatric patients with SNCD, only two out of eight showed an initial improvement in symptoms after GFD. It is likely that the diagnosis of SNCD was excluded in the remaining six subjects. ${ }^{26} \mathrm{~A}$ recent retrospective Italian study, conducted in a group of 810 patients with $\mathrm{CD}, 14$ (1.7\%) were found to be seronegative. They compared clinical, histological, and laboratory features of seronegative to seropositive patients with $\mathrm{CD}$. In particular, SNCD showed a higher prevalence $(100 \%$ vs. $34 \%, p<0.001$ ) of classic clinical manifestation, with gastrointestinal symptoms and severe villous atrophy. It resulted as a late-onset form of CD: the median age at diagnosis was significantly higher than in seropositive disease. Moreover, SNCD was described as the most frequent cause of seronegative villous atrophy $(45 \%) .{ }^{27} \mathrm{As}$ in previously published studies, our study also found a female predominance. ${ }^{27,28}$ The median age in our study was 25 (range 5-46) years, and this result was similar to a study on 12 patients with SNCD from Pakistan, which reported the median age of 31.5 years. ${ }^{28}$ Another study from Iran on 193 seropositive patients with CD also showed female predominance and a median age of 32.6 years. In this study, the most common symptom was dyspepsia (24.6\%) followed by diarrhea $(20 \%)$, anemia $(12.8 \%)$, and flatulence (7.2\%). ${ }^{29}$ However, few studies revealed late-onset SNCD, which was diagnosed in elderly people with severe malabsorption and intestinal damage. ${ }^{15,27}$ Most of the patients 11 (84.61\%) in our study had HLA DQ2 phenotype, while HLA DQ8 was demonstrated in only two (15.38\%) patients. This finding is compatible with previous studies in which the reported positivity for HLA DQ2 was $85.71 \%$ and $75 \% .{ }^{27,28}$ We found that patients with SNCD had a milder degree of villous atrophy with five (38.46\%) patients showed Marsh 2, three (23.07\%) Marsh 3a, three (23.07\%) Marsh 3b, and only two (15.38\%) patients had Marsh 3c stage. These results are similar to published studies that showed seropositive patients have a proportionately greater degree of villous atrophy than seronegative patients in various studies. ${ }^{24,30}$ However, another study is in contrast and showed SNCD is associated with severe degrees of villous atrophy. ${ }^{27}$ At the end of 6 months, 10 patients were available for follow-up, and all responded to GFD, which is similar to earlier studies. ${ }^{28,30}$ The classical clinical features, including diarrhea, abdominal pain, anemia, and weight loss, were more common in patients with SNCD compared with seropositive patients. These results are in concordance with a previous study. ${ }^{27}$ Total villous atrophy was more common in seropositive patients compared with patients with SNCD. Earlier studies also revealed that a lesser degree of villous atrophy was more common in patients with SNCD. ${ }^{15,24}$ There are few limitations to our study. First, the sample size was small. Second, some patients did not give consent for the follow-up duodenal biopsy, so we could not assess the histological improvement in these patients. Third, three of our patients were non-compliant to GFD on follow-up, so proper characterization of these patients into the spectrum of SNCD could not be agreed upon. In spite of these limitations, our study is one of the first from India that focused on a clinically important entity, which is prevalent yet frequently missed. The evaluation of seronegative villous atrophy requires a thorough workup. HLA-DQ2 and -DQ8 should be determined since a negative test rules out SNCD. ${ }^{31}$ Before starting a trial 
with GFD, other etiologies of villous atrophy should be excluded regardless of the genetic pattern. Moreover, an accurate drug review is mandatory as olmesartan is known to cause villous atrophy. ${ }^{7,11}$ Once potential causes of villous atrophy have been excluded, patients with a genetic pattern consistent with CD should start GFD. The clinical and histological responses are to be assessed to label patients as having SNCD.

In conclusion, SNCD requires recognition, as early diagnosis and treatment of these patients lead to favorable clinical outcomes. Negative serology tests must not lead to the dismissal of a CD possibility. All patients with symptoms/ signs of CD should undergo a duodenal biopsy with histopathology even if serological tests are negative. Seronegative patients require GFD to go into remission for their clinical symptoms. The diagnosis of SNCD needs to be carefully confirmed by excluding other causes of villous atrophy in order to avoid an unnecessary, lifelong GFD.

\section{ACKNOWLEDGMENTS}

1. Himanshu Sapra- Senior Project Manager, Office of Compliance \& Integrity (Ann \& Robert H. Lurie Children's Hospital of Chicago)- Statistical assistance

2. Jaya Maharshi- Typing, formatting, and technical assistance

\section{Authors' Contributions:}

Kapil Dhingra, Sandeep Ratra: Acquisition, analysis, or interpretation of the data.

Shyam Sunder Sharma, Bharat Sapra, Sandeep Nijhawan, Sudhir Maharshi: Study conception, design, and supervision, drafting of the article and critical revision for important intellectual content, final approval of the version to be published.

Sudhir Maharshi: Agreement to be accountable for all aspects of the work in ensuring that questions related to the accuracy or integrity of any part of the article are appropriately investigated and resolved.

Funding : None

\section{ETHICAL APPROVAL}

There is nothing to be declared.

\section{CONFLICT OF INTEREST}

The authors declare no conflict of interest related to this work.

\section{REFERENCES}

1. Fasano A, Catassi C. Clinical practice. Celiac disease. $N$ Engl J Med 2012;367:2419-26. doi: 10.1056/NEJMcp1113994.

2. Leffler D, Schuppan D, Pallav K, Najarian R, Goldsmith JD, Hansen J, et al. Kinetics of the histological, serological and symptomatic responses to gluten challenge in adults with coeliac disease. Gut 2013;62:996-1004. doi: 10.1136/ gutjnl-2012-302196.

3. Rubio-Tapia A, Hill ID, Kelly CP, Calderwood AH, Murray JA. ACG clinical guidelines: diagnosis and management of celiac disease. Am $J$ Gastroenterol 2013;108:656-76. doi: 10.1038/ajg.2013.79.

4. Rostami K, Kerckhaert J, Tiemessen R, Von Blomberg BM, Meijer JW, Mulder CJ. Sensitivity of antiendomysium and antigliadin antibodies in untreated celiac disease: disappointing in clinical practice. $\mathrm{Am}$ J Gastroenterol 1999;94:888-94. doi: 10.1111/j.15720241.1999.983_f.x.

5. Wahnschaffe U, Schulzke JD, Zeitz M, Ullrich R. Predictors of clinical response to gluten-free diet in patients diagnosed with diarrhea-predominant irritable bowel syndrome. Clin Gastroenterol Hepatol 2007;5:844-50. quiz 769. doi: 10.1016/j.cgh.2007.03.021.

6. Shah VH, Rotterdam H, Kotler DP, Fasano A, Green PH. All that scallops is not celiac disease. Gastrointest Endosc 2000;51:717-20. doi: 10.1067/mge.2000.104977.

7. Murray JA, Rubio-Tapia A. Diarrhoea due to small bowel diseases. Best Pract Res Clin Gastroenterol 2012;26:581-600. doi: 10.1016/j.bpg.2012.11.013.

8. Ludvigsson JF, Brandt L, Montgomery SM, Granath F, Ekbom A. Validation study of villous atrophy and small intestinal inflammation in Swedish biopsy registers. BMC Gastroenterol 2009;9:19. doi: 10.1186/1471230X-9-19.

9. Batman PA, Kotler DP, Kapembwa MS, Booth D, Christopher SP, Orenstein JM, et al. HIV enteropathy: crypt stem and transit cell hyperproliferation induces villous atrophy in HIV/microsporidia-infected jejunal mucosa. AIDS 2007;21:433-9. doi: 10.1097/ QAD.0b013e3280142ee8.

10. Malamut G, Verkarre V, Suarez F, Viallard JF, Lascaux $\mathrm{SA}, \mathrm{Cosnes} \mathrm{J}$ et al. The enteropathy associated with common variable immunodeficiency: the delineated frontiers with celiac disease. Am J Gastroenterol 2010;105:226275. doi: 10.1038/ajg.2010.214.

11. Marthey L, Cadiot G, Seksik P, Pouderoux P, Lacroute J, Skinazi $\mathrm{F}$, et al. Olmesartan-associated enteropathy:results of a national survey. Aliment Pharmacol Ther 2014;40:1103-09. doi: 10.1111/apt.12937. 
12. Malamut G, Cerf-Bensussan N, Cellier C. Identification of new cases of severe enteropathy has recently increased the spectrum of intestinal non-celiac villousatrophy. Expert Rev Gastroenterol Hepatol 2015;9:719-21. doi: 10.1586/17474124.2015.1039990.

13. Greenson JK. The biopsy pathology of non-coeliac enteropathy. Histopathology 2015;66:29-36. doi: 10.1111/ his. 12522 .

14. Makovicky P, Rimarova K, Boor A, Makovicky P, Vodicka P, Samasca G, et al. Correlation between antibodies and histology in celiac disease: Incidence of celiac disease is higher than expected in the pediatric population. Mol Med Rep 2013;8:1079-83. doi: 10.3892/ mmr.2013.1627.

15. DeGaetani M, Tennyson CA, Lebwohl B, Levis SK, Daya HA, Arguelles-Grande C, et al. Villous atrophy and negative celiac serology: a diagnostic and therapeutic dilemma. Am J Gastroenterol 2013;108:647-53. doi: 10.1038/ajg.2013.45.

16. Salmi TT, Collin P, Korponay-Szabo IR, Laurila K, Partanen J, Huhtala $\mathrm{H}$, et al. Endomysial antibody-negative coeliac disease: clinical characteristics and intestinal autoantibody deposits. Gut 2006;55:1746-53. doi: 10.1136/ gut.2005.071514.

17. Chow MA, Lebwohl B, Reilly NR, Green PH. Immunoglobin Adeficiency in celiac disease. JClin Gastroenterol 2012;46:850-4. doi: 10.1097/MCG.0b013e31824b2277.

18. Korponay-Szabo IR, Halttunen T, Szalai Z, Laurila K, Kiraly $\mathrm{R}$, Kovacs $\mathrm{JB}$, et al. In vivo targeting of intestinal and extraintestinal transglutaminase 2 by coeliac autoantibodies. Gut 2004;53:641-8. doi: 10.1136/ gut.2003.024836.

19. Lerardi E, Losurdo G, Piscitelli D, Giorgio F, Sorrentino C, Principi M, et al. Seronegative celiac disease: where is the specific setting? Gastroenterol Hepatol Bed Bench 2015;8:110-6.

20. Armstrong D, Don-Wauchope AC, Verdu EF. Testing for gluten-related disorders in clinical practice: the role of serology in managing the spectrum of gluten sensitivity. Can J Gastroenterol 2011;25:193-7. doi: $10.1155 / 2011 / 642452$

21. Rubio-Tapia A, Hill ID, Kelly CP, Calderwood AH, Murray JA. ACG clinical guidelines: diagnosis and management of celiac disease. Am J Gastroenterol 2013;108:656-76. quiz 677. doi: 10.1038/ajg.2013.79.

22. Oberhuber G, Granditsch G, Vogelsang H. The histopathology of celiac disease: time for a standardized report scheme for pathologists. Eur J Gastroenterol Hepatol 1999;11:118594. doi: 10.1097/00042737-199910000-00019.

23. Ramakrishna BS, Makharia GK, Chetri K, Dutta S, Mathur P, Ahuja V, et al. Prevalence of adult celiac disease in India: regional variations and associations. Am J Gastroenterol 2016;111:115-23. doi: 10.1038/ ajg.2015.398.

24. Abrams JA, Diamond B, Rotterdam H, Green PH. Sero- negative celiac disease: increased prevalence with lesser degrees of villous atrophy. Dig Dis Sci 2004;49:546-50. doi: 10.1023/b:ddas.0000026296.02308.00.

25. Dahele A, Kingstone K, Bode J, Anderson D, Ghosh S. Anti-endomysial antibody negative celiac disease: does additional serological testing help? Dig Dis Sci 2001;46:214-21. doi: 10.1023/a:1005589202529.

26. Ashabani A, Errabtea H, Shapan A, Tuckova L, Tlaskalova-Hogenova. Serologic markers of untreated celiac disease in Libyan children: antigliadin, antitransglutaminase, antiendomysial, and anticalreticulin antibodies. $J$ Pediatr Gastroenterol Nutr 2001;33:276-82. doi: 10.1097/00005176-200109000-00009.

27. Volta U, Caio G, Boschetti E, Giancola F, Rhoden KJ, Ruggeri E, et al. Seronegative celiac disease: Shedding light on an obscure clinical entity. Dig Liver Dis 2016;48:101822. doi: 10.1016/j.dld.2016.05.024.

28. Hanif FM, Mandhwani RK, Luck NH, Abbas Z, Mubarak M, Laeeq SM, et al. Clinicopathological study of seronegative celiac disease in adults in Pakistan : A Pilot Study. Middle East J Dig Dis 2017;9:94-9. doi: 10.15171/mejdd.2017.57.

29. Ganji A, Esmaieizadeh A, Aghayee MA, Goshayeshi L, Ghaffarzadegan K. The Clinical Presentation of Celiac Disease: Experiences from Northeastern Iran. Middle East J Dig Dis 2014;6:93-7

30. Dore MP, Pes GM, Dettori I, Vilanacci, Manca A. Realdi G. Clinical and genetic profile of patients with seronegative celiac disease: the natural history and response to gluten-free diet. BMJ Open Gastroenterol 2017;4:e00159. doi: 10.1136/bmjgast-2017-000159. eCollection 2017.

31. Ricano-Ponce I, Wijmenga C, Gutierrez-Achury J. Genetics of celiac disease. Best Pract Res Clin 2015;29:399-412. doi: 10.1016/j.bpg.2015.04.004. 